Bundesgesundheitsbl 2015 $\cdot 58: 1025$ DOI 10.1007/s00103-015-2214-6 Online publiziert: 15. August 2015

(c) Springer-Verlag Berlin Heidelberg 2015
Holger F. Rabenau ${ }^{1, *}, \%$. Norbert Bannert ${ }^{2} \cdot$ Annemarie Berger $^{1}$.

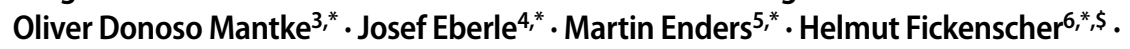
Hans-Peter Grunert ${ }^{7}$. Lutz Gürtler ${ }^{4, *}$. Albert Heim ${ }^{8, *}$. Daniela Huzly ${ }^{9, *}$.

Rolf Kaiser $^{10, \S} \cdot$ Klaus Korn ${ }^{11, *} \cdot$ Sigrid Nick ${ }^{12,{ }^{*}}$. Claudia Kücherer ${ }^{2} \cdot$ Micha Nübling $^{13, *}$. Martin Obermeier ${ }^{14, \&} \cdot$ Marcus Panning ${ }^{9, *} \cdot$ Heinz Zeichhardt ${ }^{3,15, *}, \%$

\footnotetext{
${ }^{1}$ Nationales Referenzzentrum für Retroviren, Universitätsklinikum Frankfurt

2 Robert Koch-Institut, FG 18 HIV und andere Retroviren, Berlin

3 INSTAND e.V., Gesellschaft zur Förderung der Qualitätssicherung in medizinischen Laboratorien e.V., Düsseldorf

${ }^{4}$ Max-von-Pettenkofer-Institut, Virologie, LMU München

${ }^{5}$ Labor Prof. Gisela Enders und Kollegen, MVZ GbR, Stuttgart

${ }^{6}$ Institut für Infektionsmedizin, Universitätsklinikum Schleswig-Holstein, Kiel

${ }^{7}$ GBD Gesellschaft für Biotechnologische Diagnostik mbH, Berlin

8 Institut für Virologie, Medizinische Hochschule Hannover

${ }^{9}$ Institut für Virologie, Universitätsklinikum Freiburg

${ }^{10}$ Institut für Virologie, Uniklinik Köln

${ }^{11}$ Virologisches Institut - Klinische und Molekulare Virologie, Universitätsklinikum Erlangen

${ }^{12}$ Paul-Ehrlich-Institut, Prüflabor für IVD, Langen

${ }^{13}$ Paul-Ehrlich-Institut, Molekulare Virologie, Langen

${ }^{14}$ Medizinisches Labor Dr. Thomas Berg, MVZ MIB, Berlin

${ }^{15}$ Institut für Virologie, Campus Benjamin Franklin, Charité - Universitätsmedizin Berlin

* Mitglieder der Gemeinsamen Diagnostikkommission der Deutschen Vereinigung zur Bekämpfung der Viruskrankheiten (DVV e.V.) und Gesellschaft für Virologie (GfV e.V.)

$\S$ Repräsentant der Deutschen AIDS Gesellschaft (DAIG e.V.)

\& Repräsentant der Deutschen Arbeitsgemeinschaft niedergelassener Ärzte in der Versorgung HIV-Infizierter e. V. (DAGNÄ e. V.)

\$ Repräsentant des Berufsverbandes der Ärzte für Mikrobiologie, Virologie und Infektionsepidemiologie e.V. (BÄMl e.V.)

${ }^{\%}$ Korrespondierende Autoren
}

\title{
Erratum zu: Nachweis einer Infektion mit Humanem Immundefizienzvirus (HIV): Serologisches Screening mit nachfolgender Bestätigungsdiagnostik durch Antikörper-basierte Testsysteme und/ oder durch HIV-Nukleinsäure-Nachweis
}

\author{
Stellungnahme der Gemeinsamen Diagnostikkommission der \\ Deutschen Vereinigung zur Bekämpfung von Viruskrankheiten \\ e. V. (DVV e. V.) und der Gesellschaft für Virologie e. V. (GfV e. V.)
}

\section{In Kooperation mit: Deutsche AIDS Gesellschaft e.V. (DAIG e. V.), Deutsche Arbeitsgemeinschaft niedergelassener Ärzte in der Versorgung HIV-Infizierter e. V. (DAGNÄ e. V.), Berufsverband der Ärzte für Mikrobiologie, Virologie und Infektionsepidemiologie e. V. (BÄMI e. V.)}

Erratum zu:

Bundesgesundheitsblatt

(2015) 58:877-886

DOI 10.1007/s00103-015-2174-X

In der Originalpublikation dieses Beitrags wurden die Autoren leider nicht innerhalb der Metadaten aufgeführt.
Die Liste der Autoren lautet wie folgt:

Holger F. Rabenau, Norbert Bannert, Annemarie Berger, Oliver Donoso Mantke, Josef Eberle, Martin Enders, Helmut Fickenscher, Hans-Peter Grunert, Lutz Gürtler, Albert Heim, Daniela Huzly, Rolf Kaiser, Klaus Korn, Sigrid Nick, Claudia Kücherer, Micha Nübling, Martin Ober- meier, Marcus Panning, Heinz Zeichhardt.

Wir bitten den Fehler zu entschuldigen.

Der Verlag

Die Onlineversion des Originalbeitrages ist unter doi:10.1007/s00103-015-2174-x zu finden. 